\title{
Predictors of post-stroke body temperature elevation
}

\author{
Rebecca Ruborg ${ }^{1}$, Karin Gunnarsson ${ }^{1}$ and Jakob O. Ström ${ }^{1,2,3^{*}}$ (D)
}

\begin{abstract}
Background: Growing evidence indicates that elevated body temperature after stroke is associated with unfavorable outcome. The aim of the current study was to investigate which factors predict temperature elevation within $48 \mathrm{~h}$ of stroke onset. Specifically, we hypothesized that temperature elevation would be associated with stroke symptom severity and that hemorrhagic stroke would cause a more pronounced temperature increase compared to ischemic stroke.

Methods: The medical records of 400 stroke patients were retrospectively reviewed. Multiple linear regression analysis was used to determine which factors were associated with elevated body temperature.

Results: Several factors were significantly associated with peak body temperature (the highest recorded body temperature) within $48 \mathrm{~h}$ of stroke onset: stroke severity measured by the National Institutes of Health Stroke Scale (NIHSS) (regression coefficient; (RC) 0.022), female gender (RC 0.157), tympanic/non-rectal temperature reading (RC -0.265 ), swallowing difficulties (RC 0.335), intubation (RC 0.470), antipyretic treatment (RC 0.563), and C-reactive protein $>50$ or signs of infection at admission (RC 0.298). Contrary to our expectations, patients with intracerebral hemorrhage did not have higher peak body temperatures than patients with ischemic stroke.

Conclusions: In conclusion, temperature elevation within the first $48 \mathrm{~h}$ of stroke onset is common, can be partially predicted using information at admission and is strongly associated with stroke severity. The strong association with stroke severity may, at least partly, explain the previously described association between post-stroke temperature elevation and unfavorable outcome.
\end{abstract}

Keywords: Stroke, Fever, Body temperature, Infection

\section{Background}

Different factors have been shown to be associated with recovery from stroke, for example body temperature after the incident [1-3]. In addition to being at risk of bacterial infections such as pneumonia, stroke patients often suffer from temperature elevations without an identifiable infection; possibly endogenous fever due to direct effects of the infarction or hemorrhage on the brain [4-6]. Although numerous studies regarding body temperature after stroke have been published, treatment guidelines for post-stroke temperature elevation are

\footnotetext{
* Correspondence: jakob.strom@oru.se

'Department of Neurology, Faculty of Medicine and Health, Örebro University, Örebro, Sweden

${ }^{2}$ Department of Clinical Chemistry and Department of Clinical and Experimental Medicine, Linköping University, Linköping, Sweden Full list of author information is available at the end of the article
}

ambiguous and no firm clinical consensus has been reached [7].

A profound understanding of the pathophysiology of body temperature elevation after stroke onset is pivotal for the development of successful treatment strategies. Clues regarding endogenous temperature elevation are especially important, since distinguishing between endogenous and infectious fever is a common clinical dilemma. One important piece of this puzzle could be provided by mapping factors that predispose to temperature elevation. For example, if it was known that patients with intracerebral hemorrhage are more prone to early temperature elevation compared to patients with ischemic stroke, then this could indicate that the elevation in temperature is partly mediated by the intrathecal release of blood products and that higher non-infectious temperature elevations are to be expected in these 
patients. Previously, stroke severity, as well as various patient characteristics such as age and gender, has been shown to be associated with post-stroke temperature elevation $[1,8]$. However, several important questions, such as whether hemorrhagic or ischemic stroke causes the most temperature elevation, remain unanswered.

Therefore, the aim of this study was to investigate which factors were associated with peak body temperature (defined as the highest recorded body temperature for each patient) within $48 \mathrm{~h}$ of stroke onset. Specifically, we hypothesized that peak body temperature would be associated with stroke severity and that hemorrhagic stroke would cause a more pronounced temperature increase compared to ischemic stroke.

\section{Methods}

This study was a retrospective review of the medical records at the University Hospital in Örebro. A medical secretary collected the names of patients diagnosed with International Classification of Diseases (ICD) I61 and I63 from the Swedish Stroke Register during 2014 and 2015. Inclusion criteria were cerebral infarction or intracerebral hemorrhage together with at least one temperature reading within the first $48 \mathrm{~h}$ of clinical stroke onset (median number of temperature registrations per patient and day was 3 ). Altogether, 400 patients with ischemic or hemorrhagic stroke were included from August 2014 to December 31st, 2015. Patients with transient symptoms within $24 \mathrm{~h}$ were assessed as transient ischemic attack (TIA) and were excluded, even if there were signs of ischemia on computed tomography or if treatment with thrombolysis had been given. A flow chart of the inclusion process is presented in Fig. 1.

Data including patient characteristics, stroke subtype, laboratory parameters, previous and newly initiated treatments and temperature readings was extracted from the medical records in accordance with a prewritten protocol (all temperature readings during $48 \mathrm{~h}$ were noted and the highest temperature readings within time interval were used in the analyses). If smoking was explicitly mentioned in the medical record, the patient was classified as a smoker; if no smoking was reported or smoking was not mentioned, it was assumed that the patient did not smoke. If the exact time of the stroke onset was uncertain, the time-point at which the patient had last been seen free from symptoms was considered as stroke onset. In cases where exact timing of stroke onset was lacking, time-points were stipulated according to a prespecified algorithm: symptom onset described as in the "morning", "afternoon" and "evening" was interpreted as stroke onset at $8 \mathrm{am}$, $4 \mathrm{pm}$, and $8 \mathrm{pm}$, respectively. For patients who had woken up with stroke symptoms with no recorded time of going to bed, stroke onset was assumed to have been at $10 \mathrm{pm}$. If the patients had had a National Institutes of Health Stroke

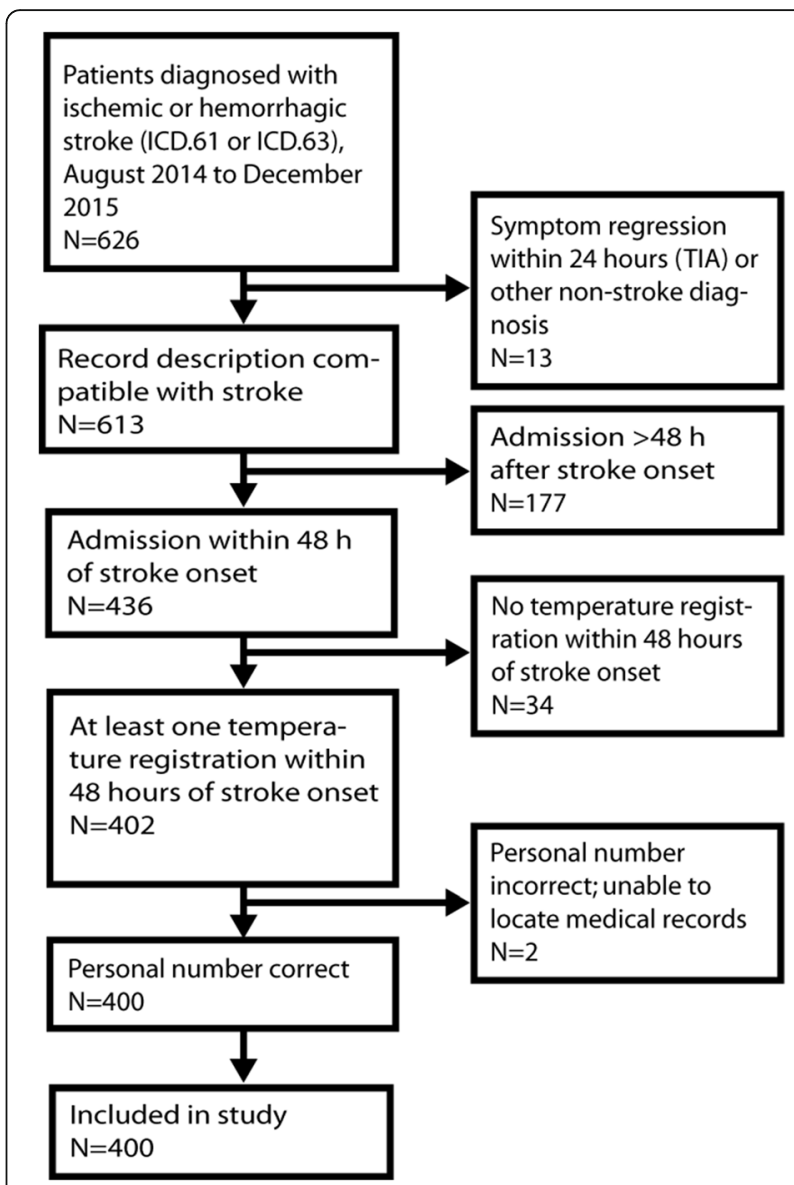

Fig. 1 Flow chart showing the inclusion process. ICD = International Classification of Diseases and Related Health Problems; TIA = transient ischemic attack

Scale (NIHSS) score documented at arrival, the score was noted, whereas in patients without documented NIHSS score, neurological status was converted to NIHSS score manually in accordance with a protocol. Where a patient had suffered from stroke earlier and had residual symptoms that were described in the medical records, this was subtracted from the current NIHSS score to get an estimation of the severity of the current stroke. Where hypertensive medication or statins had been prescribed during hospitalization, these were regarded as signs of hypertension or hyperlipidemia. At the intensive care unit, temperature had been measured using a urinary catheter, which is comparable to rectal temperature measurements, and regarded as such in the statistical analysis. If a new stroke had occurred during hospital stay, the second event was excluded from the study. C-reactive protein (CRP) more than 50 was counted as a sign of infection.

\section{Statistics}

Normally distributed data was presented with mean values and standard deviation (SD). Median, minimum 
and maximum values and/or interquartile range (IQR) were used for data not normally distributed. Frequencies were calculated for all categorical variables. Peak body temperature was defined for each patient as the highest recorded temperature within $48 \mathrm{~h}$ from stroke onset. Multiple linear regression analyses were performed using SPSS 23 (IBM Statistics 23 Armonk, NY, USA), assessing the effect of patient characteristics and stroke characteristics (including hemorrhagic/ischemic and NIHSS) on the outcome variable peak body temperature (treated as a continuous variable). Backward multiple regression analysis was performed using a $p$-value $>0.2$ to exclude non-significant variables from the regression model. A $\mathrm{p}$-value $<0.05$ was considered statistically significant in the final regression analysis.

\section{Ethics}

This study was a retrospective review of medical records at the University Hospital in Örebro and was performed with approval from the regional ethical committee in Uppsala, Sweden (2015/516). Written consent from the patients or their relatives to participate in the study was not obtained. However, when being registered with the Swedish Stroke Register, patients are informed of the possibility that the data could be used in studies, and of their right to withdraw their data from the register. The same 400 patients included here were also included in a study on predicting factors for mortality after stroke, simultaneously submitted for publication.

\section{Results}

In total, 626 patients with cerebral infarction or intracerebral hemorrhage were reported by Örebro University Hospital to the Swedish Stroke Register between August 2014 and December 2015. Of these, 400 fulfilled the inclusion criteria and 226 were excluded. Patients with symptom regression within $24 \mathrm{~h}$ or a diagnosis that was not ischemic or hemorrhagic stroke were excluded as well as patients who arrived to the hospital more than $48 \mathrm{~h}$ after stroke onset, patients with no temperature reading within $48 \mathrm{~h}$ of stroke onset and patients whose medical records could not be found (Fig. 1). Baseline characteristics are summarized in Table 1.

The mean of all patients' peak body temperature readings within $48 \mathrm{~h}$ was $37.5{ }^{\circ} \mathrm{C}$, the highest peak body temperature was $41.5{ }^{\circ} \mathrm{C}$ and the lowest peak body temperature was $34.9{ }^{\circ} \mathrm{C}$. Temperature subgroups, signs of infection and other hospitalization parameters are summarized in Table 2. The median frequency/number of temperature readings per patient and day within $48 \mathrm{~h}$ of stroke onset was three.

In the multiple linear regression analysis, NIHSS score, female gender, rectal (as opposed to tympanic) temperature, positive swallowing test (indicating
Table 1 Baseline characteristics

\begin{tabular}{ll}
\hline Age, yrs. mean \pm SD & $77 \pm 12.5$ \\
Women, N (\%) & $205(51)$ \\
Smoking, N (\%) & $47(12)$ \\
Anticoagulants at admission, N (\%) & $53(13)$ \\
Antithrombotic therapy at admission, N (\%) & $145(36)$ \\
Antipyretic drugs daily at admission, N (\%) & $91(23)$ \\
Antipyretic drugs, when needed, at & $30(8)$ \\
admission, N (\%) & $17(4)$ \\
Antibiotics at admission, N (\%) & $298(75)$ \\
Hypertension, N (\%) & $90(23)$ \\
Diabetes, N (\%) & $129(32)$ \\
Atrial fibrillation, N (\%) & $210(53)$ \\
Hypercholesterolemia, N (\%) & $132(33)$ \\
Previous stroke/TIA, N (\%) & $40(10)$ \\
Malignancy, N (\%) & $101(25)$ \\
Ischemic coronary heart disease/heart & \\
failure, N (\%) & $41(10)$ \\
Chronic obstructive pulmonary disease/ \\
other chronic lung disease, N (\%) \\
Chronic inflammatory disease, N (\%) \\
Chronic infection, N (\%)
\end{tabular}

SD standard deviation, TIA transient ischemic attack

dysphagia), intubation, $C R P>50 /$ signs of infection at admission, and antipyretic treatment within $48 \mathrm{~h}$ were positively associated with peak body temperature within $48 \mathrm{~h}$. These variables are presented in Table 3, together with the variables that remained in the last step of the regression analysis. Variables excluded from the preceding backward multiple regression analysis are presented in Table 4, in the order of exclusion. Rsquared $\left(\mathrm{r}^{2}\right)$ was 0.355 for the entire multiple- regression model.

\section{Discussion}

In this retrospective study we confirm that body temperature elevation within $48 \mathrm{~h}$ of stroke onset is common and associated with neurological status at admission (NIHSS score), female gender, swallowing difficulties, intubation and CRP $>50$ or signs of infection at admission.

As hypothesized, in the current study, symptom severity as determined by NIHSS score was a strong predictor of increased body temperature, which is also in concordance with several previous studies [1,9-11]. This association could have several explanations that are not mutually exclusive. Firstly, it is possible that the initial elevation of body temperature is partly non-infectious; either due to local effects on cerebral thermoregulatory centers or to stimulation of the immune system caused by necrosis, as would be expected with a necrotic process in any organ $[6,12]$. If local effects on thermoregulatory centers were responsible for the elevation in 
Table 2 Parameters during hospitalization

\begin{tabular}{|c|c|}
\hline \multicolumn{2}{|l|}{ NIHSS score at admission, $N(\%)$} \\
\hline $0 ; N(\%)$ & $54(14)$ \\
\hline $1-4 ; N(\%)$ & $185(46)$ \\
\hline $5-15 ; N(\%)$ & $128(32)$ \\
\hline $16-20 ; N(\%)$ & $9(2)$ \\
\hline$>21 ; N(\%)$ & $24(6)$ \\
\hline NIHSS score at admission, median (IQR) & $3(7)$ \\
\hline Antibiotics at admission, $N(\%)$ & $17(4)$ \\
\hline Temperature at admission, ${ }^{\circ} \mathrm{C}$, mean $\pm \mathrm{SD}$ & $36.5 \pm 3.8$ \\
\hline CRP level at admission, mg/L, median (min- max) & $3.5(1-194)$ \\
\hline Signs of infection or CRP > 50 at admission, $N(\%)$ & $65(16)$ \\
\hline \multicolumn{2}{|l|}{ Stroke type, $N(\%)$} \\
\hline Infarction & $351(88)$ \\
\hline Hemorrhage & $49(12)$ \\
\hline $\begin{array}{l}\text { Number of temperature readings median } \\
\text { (min-max) }\end{array}$ & $3(1-27)$ \\
\hline \multicolumn{2}{|l|}{ Peak body temperature within 48 h, N (\%) } \\
\hline$<35.5^{\circ} \mathrm{C}$ & $1(0.3)$ \\
\hline $35.6-36.5^{\circ} \mathrm{C}$ & $24(6)$ \\
\hline $36.6-37.5^{\circ} \mathrm{C}$ & $198(50)$ \\
\hline $37.6-38.5^{\circ} \mathrm{C}$ & $140(35)$ \\
\hline $38.6-39.5^{\circ} \mathrm{C}$ & $32(8)$ \\
\hline $39.6-40.5^{\circ} \mathrm{C}$ & $5(1)$ \\
\hline $40.6-41.5^{\circ} \mathrm{C}$ & $1(0.3)$ \\
\hline \multicolumn{2}{|l|}{ Temperature measurement method, $N(\%)$} \\
\hline Rectal & $263(66)$ \\
\hline Tympanic & $137(34)$ \\
\hline Thrombolysis, N (\%) & $23(6)$ \\
\hline Thrombectomy, N (\%) & $2(0.5)$ \\
\hline Decompression surgery, $N(\%)$ & $2(0.5)$ \\
\hline $\begin{array}{l}\text { Antipyretic treatment within } 48 \mathrm{~h} \text { of stroke } \\
\text { onset, } N(\%)\end{array}$ & $201(50)$ \\
\hline Antibiotic treatment during hospital stay, $N(\%)$ & $63(16)$ \\
\hline CRP > 50 during hospitalization, $N(\%)$ & $34(9)$ \\
\hline Indwelling urinary catheter during hospitalization, $N(\%)$ & $82(21)$ \\
\hline Positive swallowing test, $N(\%)$ & $43(11)$ \\
\hline Undergoing intubation, N (\%) & $12(3)$ \\
\hline $\begin{array}{l}\text { Signs of urinary tract infection- /positive urine cultures, } \\
N(\%)\end{array}$ & $31(8)$ \\
\hline Signs of pneumonia or positive lung radiology, $N(\%)$ & $12(3)$ \\
\hline Positive nasopharyngeal cultures, N (\%) & $4(1)$ \\
\hline Positive blood cultures, N (\%) & $0(0)$ \\
\hline
\end{tabular}

CRP C-reactive protein, IQR interquartile range, NIHSS National Institutes of Health Stroke Scale, SD standard deviation temperature, an association between stroke location and temperature elevation would be expected [6]. Unfortunately, such analyses could not be performed on the current data because of the inclusion of a large number of patients with ischemic stroke, in many of whom exact lesion location was unknown. A second possibility is that the stroke makes the patient prone to develop infections and that this effect is more pronounced with larger lesions. In a previous study, the NIHSS score was shown to predict post-stroke infections [13]. Stroke is known to make the patient susceptible to infections by several mechanisms, including immunosuppression [14], dysphagia [15] and urinary incontinence, prompting use of urinary catheters [16]. The strong relation between stroke severity and post-stroke body temperature elevation may explain in part the previously described association between temperature and stroke outcome, and emphasizes the importance of meticulous confounder control when investigating such relations.

The second hypothesis, that intracerebral hemorrhage would cause more pronounced temperature elevations than ischemic stroke, was not corroborated. We assumed that initial temperature elevation would mainly be due to local effects of the brain lesion, and that mechanisms for non-infectious temperature elevation caused by the lesion's direct effects on thermoregulation in the brain would differ between ischemia and hemorrhage [5, 17, 18]. Furthermore, there is a widespread notion among stroke physicians that hemorrhagic stroke patients are more prone to fever. Surprisingly, in our study population, whether the patient had ischemia or hemorrhage was not associated to peak body temperature within $48 \mathrm{~h}$ of stroke onset. In other words, patients with hemorrhage did not have higher temperature than patients with ischemic stroke after controlling for the other factors. The factor was excluded in the preceding backward analysis with a $p$ value of 0.647 , which in combination with the large study sample indicates that the risk for a type II error was very low. Previous studies investigating stroke severity and its correlation to post-stroke events either included ischemic or hemorrhagic stroke, or did not adequately control for stroke severity, and therefore this question has hitherto been left unanswered $[1,18]$. The current lack of association between stroke subtype and temperature does not allow conclusions to be drawn regarding the pathophysiology of post-stroke body temperature .

Several other observations were made in the current study, which are, however, unrelated to the main hypothesis and therefore should be interpreted with caution. Firstly, an increased susceptibility to elevation in temperature in females was noted, in line with Saini et al. who observed that female gender was an independent predictor of elevated temperature within $24 \mathrm{~h}$ [1]. This could be explained by differences in temperature 
Table 3 Final results of the multiple linear regression analysis

\begin{tabular}{|c|c|c|c|c|}
\hline & Regression coefficient & 95\% confidence interval & $P$-value & VIF \\
\hline (Constant) & 37.081 & $36.929-37.232$ & 0.000 & \\
\hline NIHSS score & 0.022 & $0.010-0.034$ & 0.000 & 1.386 \\
\hline Antipyretic treatment within $48 \mathrm{~h}$ & 0.563 & $0.432-0.694$ & 0.000 & 1.120 \\
\hline Tympanic/Non rectal temperature reading & -0.265 & $-0.401--0.129$ & 0.000 & 1.087 \\
\hline CRP $>50$ or signs of infection at admission & 0.298 & $0.122-0.473$ & 0.001 & 1.090 \\
\hline Unknown CRP level without signs of infection & 0.047 & $-0.298-0.392$ & 0.789 & 1.047 \\
\hline Positive swallowing test & 0.335 & $0.130-0.540$ & 0.001 & 1.054 \\
\hline Female gender & 0.157 & $0.031-0.284$ & 0.014 & 1.036 \\
\hline Intubation & 0.470 & $0.044-0.896$ & 0.031 & 1.379 \\
\hline Diabetes & 0.142 & $-0.009-0.293$ & 0.065 & 1.037 \\
\hline Smoking & -0.175 & $-0.371-0.020$ & 0.079 & 1.037 \\
\hline Right sided stroke & -0.087 & $-0.222-0.048$ & 0.204 & 1.162 \\
\hline Hemisphere not known & 0.037 & $-0.163-0.238$ & 0.714 & 1.171 \\
\hline
\end{tabular}

CRP C-reactive protein, NIHSS National Institutes of Health Stroke Scale, VIF variance inflation factor

Significant $p$-values $(<0.05)$ are written in boldface

regulation between the sexes, since women generally seem to have a higher body temperature [19]. In the current regression analysis, a positive swallowing test and intubation were associated with peak body temperature within $48 \mathrm{~h}$, which seems reasonable given the previously known connection between swallowing difficulties and respiratory infections after stroke onset $[15,20]$. Furthermore, in the current study, age was surprisingly not associated with increased temperature. This is in contrast to Yan et al. who claimed that age $>65$ predisposes for post-stroke fever [8]. Further, Ribeiro et al. concluded that NIHSS score and high age are correlated

Table 4 Variables excluded from the preceding backward multiple linear regression analysis, in order of exclusion

\begin{tabular}{ll}
\hline Variable & $P$-value at exclusion \\
\hline Chronic infection & 0.887 \\
Hypertension & 0.872 \\
Unknown CRP level without signs of infection & 0.848 \\
Thrombolysis & 0.822 \\
Hyperlipidemia & 0.783 \\
Coronary heart disease/heart failure & 0.800 \\
Hemisphere not reported & 0.709 \\
Infarction & 0.647 \\
Chronic inflammatory disease & 0.630 \\
Previous stroke/TIA & 0.355 \\
Chronic obstructive pulmonary disease/ & 0.267 \\
Other chronic lung disease & \\
Malignancy & 0.264 \\
Age & 0.210 \\
Atrial fibrillation & 0.228 \\
\hline
\end{tabular}

CRP C-reactive protein, TIA transient ischemic attack to post-stroke pneumonia [21]. A possible explanation for the current findings is that although elderly patients have a higher risk for post-stroke infections, their generally lower body temperature [22] might compensate for possible temperature elevations due to infections.

The study has some limitations that need to be emphasized. Several patients were excluded due to temperature readings more than $48 \mathrm{~h}$ from stroke onset or unknown timing of stroke onset, which might imply that the cohort is less representative for the study population. Another important limitation of this retrospective study regards determining patients' peak temperature. According to the inclusion criteria, at least one temperature reading within $48 \mathrm{~h}$ of stroke onset was required for inclusion. However, because measurements were generally not performed more than three times a day, and in some patients only once daily, the recorded temperature readings may not reflect the patients' temperature peaks. Another weakness of the study is that only in a few cases was neurologic status noted as NIHSS score directly by the examiner. The majority of the NIHSS scores were instead retrospectively calculated to NIHSS based on the description of neurologic status in the patient records, which is a source of error even if performed in accordance with a strict protocol. Previous disability was also retrospectively computed and subtracted from the total NIHSS to achieve NIHSS for the current stroke, which, consequently might be a weakness since it requires careful documentation in the medical records. In an effort to separate non-infectious temperature elevation from temperature elevation with infectious etiology, signs of infection during hospitalization were summarized in Table 2 . The majority of patients with positive signs of infection and 
treatment with antibiotics had temperature elevation within $48 \mathrm{~h}$. However, only a minority of patients with temperature elevations were subjected to lung radiology, blood cultures, or other investigations to pinpoint infections, making our estimate of the rate of infections extremely uncertain. Previous studies indicate that poststroke infections occur between 1 and 7 days after stroke onset $[9,15,23]$; in the present study, few patients received antibiotics within the first $48 \mathrm{~h}$ from admission (median time to antibiotic treatment was 3 days). As well as weaknesses, the current study also has several strengths. First of all, the in-depth evaluation of the medical records of a relatively large patient cohort can be considered a strength. Further, the multiple regression analysis makes it possible to adjust for confounding factors. Although predicting factors for post-stroke temperature elevation have been investigated in several previous studies, few have focused on factors associated with body temperature within $48 \mathrm{~h}$ of both ischemic and hemorrhagic stroke.

\section{Conclusions}

In accordance with the initial hypothesis, post-stroke temperature elevation seems to be associated with NIHSS status at admission as well as with gender, swallowing difficulties, intubation and CRP $>50$ or signs of infection at admission, but not with hemorrhage or ischemic stroke. The strong association with stroke severity may, at least partly, explain the association between post-stroke temperature elevation and less favorable outcome seen in previous studies [1-3].

\section{Abbreviations \\ CRP: C-reactive protein; ICD: International classification of diseases and related health problems; IQR: Interquartile range; NIHSS: National institute of health stroke scale; RC: Regression coefficient; SD: Standard deviation; \\ TIA: Transient ischemic attack; VIF: Variance inflation factor}

\section{Acknowledgements}

We gratefully acknowledge the assistance of Marie Lokander at Region Örebro län. The study was funded by Region Örebro län.

\section{Funding}

The study was funded by Örebro County Council (Region Örebro län).

\section{Availability of data and materials}

The data used in the current study is patient- related, and can therefore not be made public

\section{Authors' contributions}

RR extracted all data from the medical records, performed most of the analyses, and drafted the manuscript. All authors (RR, KG and JOS) contributed to the study design, revised the manuscript and approved the final version before submission.

\section{Ethics approval and consent to participate}

Before data collection, the study protocol was approved by the Regional Ethical Review Board of Uppsala (2015/516). The study was performed in accordance with relevant guidelines and regulations. Written consent from the patients or their relatives for participation in the study was not obtained, but at the time the patients were registered in the Swedish Stroke Register, they were informed of the possibility that the data might be used in studies and of their right to withdraw their data from the register.

Consent for publication

Not applicable.

\section{Competing interests}

Jakob O Ström has received a consultant fee for participation on an Advisory Board of Bayer AB in 2016. The other authors have nothing to disclose.

\section{Publisher's Note}

Springer Nature remains neutral with regard to jurisdictional claims in published maps and institutional affiliations.

\section{Author details}

${ }^{1}$ Department of Neurology, Faculty of Medicine and Health, Örebro University, Örebro, Sweden. ${ }^{2}$ Department of Clinical Chemistry and Department of Clinical and Experimental Medicine, Linköping University, Linköping, Sweden. ${ }^{3}$ Region Örebro Län, Neuro- och rehabmedicinska kliniken, Södra Grev Rosengatan, 70185 Örebro, Sweden.

Received: 12 May 2017 Accepted: 5 December 2017

Published online: 13 December 2017

References

1. Saini M, Saqqur M, Kamruzzaman A, Lees KR, Shuaib A, Investigators V. Effect of hyperthermia on prognosis after acute ischemic stroke. Stroke. 2009:40(9):3051-9.

2. Azzimondi G, Bassein L, Nonino F, Fiorani L, Vignatelli L, Re G, D'Alessandro R. Fever in acute stroke worsens prognosis. A prospective study. Stroke. 1995;26(11):2040-3.

3. Popovic N, Stefanovic-Budimkic M, Mitrovic N, Urosevic A, Milosevic B, Pelemis M, Jevtovic D, Beslac-Bumbasirevic L, Jovanovic D. The frequency of poststroke infections and their impact on early stroke outcome. J Stroke Cerebrovasc Dis. 2013;22(4):424-9.

4. Reith J, Jorgensen HS, Pedersen PM, Nakayama H, Raaschou HO, Jeppesen LL, Olsen TS. Body temperature in acute stroke: relation to stroke severity, infarct size, mortality, and outcome. Lancet. 1996;347(8999):422-5.

5. Steiner AA, Branco LG. Central CO-heme oxygenase pathway raises body temperature by a prostaglandin-independent way. J Appl Physiol (1985). 2000;88(5):1607-13.

6. Sung $\mathrm{CY}$, Lee $\mathrm{TH}$, Chu NS. Central hyperthermia in acute stroke. Eur Neurol. 2009;62(2):86-92.

7. Ntaios G, Dziedzic T, Michel P, Papavasileiou V, Petersson J, Staykov D, Thomas B, Steiner T, European Stroke O. European stroke organisation (ESO) guidelines for the management of temperature in patients with acute ischemic stroke. Int J Stroke. 2015;10(6):941-9.

8. Yan F, Zhang $D, X u H$, Guo H. Risk factors for fever in critically ill patients with acute new-onset stroke. Neurol Res. 2008;30(4):394-9.

9. Grau AJ, Buggle F, Schnitzler P, Spiel M, Lichy C, Hacke W. Fever and infection early after ischemic stroke. J Neurol Sci. 1999;171(2):115-20.

10. Li G, Xu XY, Wang Y, Gu XB, Xue YY, Zuo L, Yu JM. Mild-to-moderate neurogenic pyrexia in acute cerebral infarction. Eur Neurol. 2011;65(2): 94-8.

11. Wong AA, Davis JP, Schluter PJ, Henderson RD, O'Sullivan JD, Read SJ. The time course and determinants of temperature within the first $48 \mathrm{~h}$ after ischaemic stroke. Cerebrovasc Dis. 2007;24(1):104-10.

12. Kacprzak M, Kidawa M, Zielinska M. Fever in myocardial infarction: is it still common, is it still predictive? Cardiol J. 2012;19(4):369-73.

13. Almeida SR, Bahia MM, Lima FO, Paschoal IA, Cardoso TA, Li LM. Predictors of pneumonia in acute stroke in patients in an emergency unit. Arq Neuropsiquiatr. 2015;73(5):415-9.

14. Klehmet J, Harms H, Richter M, Prass K, Volk HD, Dirnagl U, Meisel A, Meisel C. Stroke-induced immunodepression and post-stroke infections: lessons from the preventive antibacterial therapy in stroke trial. Neuroscience. 2009;158(3): 1184-93.

15. Brogan E, Langdon C, Brookes K, Budgeon C, Blacker D. Dysphagia and factors associated with respiratory infections in the first week post-stroke. Neuroepidemiology. 2014;43(2):140-4. 
16. Poisson SN, Johnston SC, Josephson SA. Urinary tract infections complicating stroke: mechanisms, consequences, and possible solutions. Stroke. 2010;41(4):e180-4.

17. Dietrich WD, Halley M, Valdes I, Busto R. Interrelationships between increased vascular permeability and acute neuronal damage following temperature-controlled brain ischemia in rats. Acta Neuropathol. 1991;81(6): $615-25$

18. Honig A, Michael S, Eliahou R, Leker RR. Central fever in patients with spontaneous intracerebral hemorrhage: predicting factors and impact on outcome. BMC Neurol. 2015;15:6.

19. Kim H, Richardson C, Roberts J, Gren L, Lyon JL. Cold hands, warm heart. Lancet. 1998;351(9114):1492.

20. Brogan E, Langdon C, Brookes K, Budgeon C, Blacker D. Can't swallow, can't transfer, can't toilet: factors predicting infections in the first week poststroke. J Clin Neurosci. 2015;22(1):92-7.

21. Ribeiro PW, Cola PC, Gatto AR, da Silva RG, Luvizutto GJ, Braga GP, Schelp AO, de Arruda Henry MA, Bazan R. Relationship between Dysphagia, National Institutes of Health stroke scale score, and predictors of pneumonia after ischemic stroke. J Stroke Cerebrovasc Dis. 2015;24(9):2088-94

22. Roghmann MC, Warner J, Mackowiak PA. The relationship between age and fever magnitude. Am J Med Sci. 2001;322(2):68-70

23. Maeshima S, Osawa A, Hayashi T, Tanahashi N. Elderly age, bilateral lesions, and severe neurological deficit are correlated with stroke-associated pneumonia. J Stroke Cerebrovasc Dis. 2014;23(3):484-9.

\section{Submit your next manuscript to BioMed Central} and we will help you at every step:

- We accept pre-submission inquiries

- Our selector tool helps you to find the most relevant journal

- We provide round the clock customer support

- Convenient online submission

- Thorough peer review

- Inclusion in PubMed and all major indexing services

- Maximum visibility for your research

Submit your manuscript at www.biomedcentral.com/submit 